\title{
Applications of Nanomaterials in Biology and Medicine
}

\author{
Lifeng Dong, ${ }^{1,2}$ Michael M. Craig, ${ }^{3}$ Dongwoo Khang,, ${ }^{4}$ and Chunying Chen ${ }^{5}$ \\ ${ }^{1}$ College of Materials Science and Engineering, Qingdao University of Science and Technology, Qingdao 266042, China \\ ${ }^{2}$ Department of Physics, Astronomy, and Materials Science, Missouri State University, Springfield, MO 65897, USA \\ ${ }^{3}$ Department of Biomedical Sciences, Missouri State University, Springfield, MO 65897, USA \\ ${ }^{4}$ School of Nano and Advanced Materials Engineering, Gyeongsang National University, Gyeongnam 660-701, Republic of Korea \\ ${ }^{5}$ National Center for Nanoscience and Technology, Beijing 100190, China
}

Correspondence should be addressed to Lifeng Dong, lifengdong@missouristate.edu

Received 17 May 2012; Accepted 17 May 2012

Copyright (C) 2012 Lifeng Dong et al. This is an open access article distributed under the Creative Commons Attribution License, which permits unrestricted use, distribution, and reproduction in any medium, provided the original work is properly cited.

Due to nanoscale effects and increased surface area, nanomaterials have been investigated as promising tools for the advancement of diagnostic biosensors, drug and gene delivery, and biomedical imaging. In comparison to their larger counterparts, nanomaterials have unique physicochemical and biological properties. Many properties of nanomaterials, such as size, shape, chemical composition, surface structure and charge, aggregation and agglomeration, and solubility, can greatly influence their interactions with biomolecules and cells. For example, nanoparticles with size-tunable light emission have been employed to produce exceptional images of tumor sites; single-walled carbon nanotubes, having diameters comparable to the width of DNA molecules, have demonstrated an impressive potential as high-efficiency delivery transporters for biomolecules into cells. Therefore, the main emphasis of this special issue focuses on the development of some nanomaterials and their applications in biology and medicine.

This special issue contains three review articles and six research articles. Among the review articles, T. Kaur et al. examine and discuss bacteriophage nanomedicine applications and the immunomodulator effects of bacteriophage exposure and treatment modalities in paper titled "Immunocompatibility of bacteriophages as nanomedicines." K. J. Flores et al. highlight the literature relating to gene-based vaccines, especially DNA vaccines, and explore the combination of RNA and nanomaterials (e.g., gold nanoparticles, nanoliposomes, and dendrimers) for prevention and treatment of disease in paper titled "Tipping the proteome with genebased vaccines: weighing in on the role of nanomaterials." Z. Zhang and C. Huang summarize recent developments in the field of intra-articular drug delivery systems using micro/nanocarriers, such as polymeric micro/nanoparticles, liposomes, and hydrogels, in paper titled "Micro- and nanocarrier mediated intra-articular drug delivery systems for the treatment of osteoarthritis."

Among the six research articles, four articles are related to the study of carbon nanotubes. M. Mananghaya et al. use density functional theory to investigate the solubility and reactivity of single-walled carbon nanotubes (SWCNTs) in paper titled "Theoretical investigation on the solubilization in water of functionalized single-wall carbon nanotubes." H. Haniu et al. report effects of graphitization temperature and diameter of multiwalled carbon nanotubes on their biological responses, such as cell viability, total reactive oxygen, and/or nitrogen species productions, and cytokine secretion in paper titled "Manufacturing strategy for multiwalled carbon nanotubes as a biocompatible and innovative material." J. Harvey et al. show that SWCNTs facilitate DNA delivery into microvascular endothelial cells, even their nuclei, thus suggesting that carbon nanotubes have therapeutic potential as drug and gene vehicles in paper titled "Uptake of single-walled carbon nanotubes conjugated with DNA by microvascular endothelial cells." L. Dong et al. demonstrate SWCNTs' antibacterial properties against Salmonella enterica and Escherichia coli, indicating that carbon nanotubes could become an effective alternative to antibiotics in dealing with drug-resistant and multidrugresistant bacterial strains in paper titled "Antimicrobial activity of single-walled carbon nanotubes suspended in different surfactants." S. Anuchapreeda et al. develop a new formulation of a curcumin lipid nanoemulsion of $47-55 \mathrm{~nm}$ 
for cancer chemotherapy in paper titled "Preparation of lipid nanoemulsions incorporating curcumin for cancer therapy." R. K. DeLong et al. comparatively characterize nucleic acid conjugates with protamine and gold nanoparticles in the nano range of concentrations by UV/Vis spectrum, dynamic laser light scattering, fluorimetry, and gel electrophoresis in paper titled "Biomolecular triconjugates formed between gold, protamine, and nucleic acid: comparative characterization on the nanoscale."

Lastly, the editors would like to acknowledge and thank all authors for their contributions to this special issue and the reviewers for their time and dedication.

Lifeng Dong

Michael M. Craig

Dongwoo Khang

Chunying Chen 

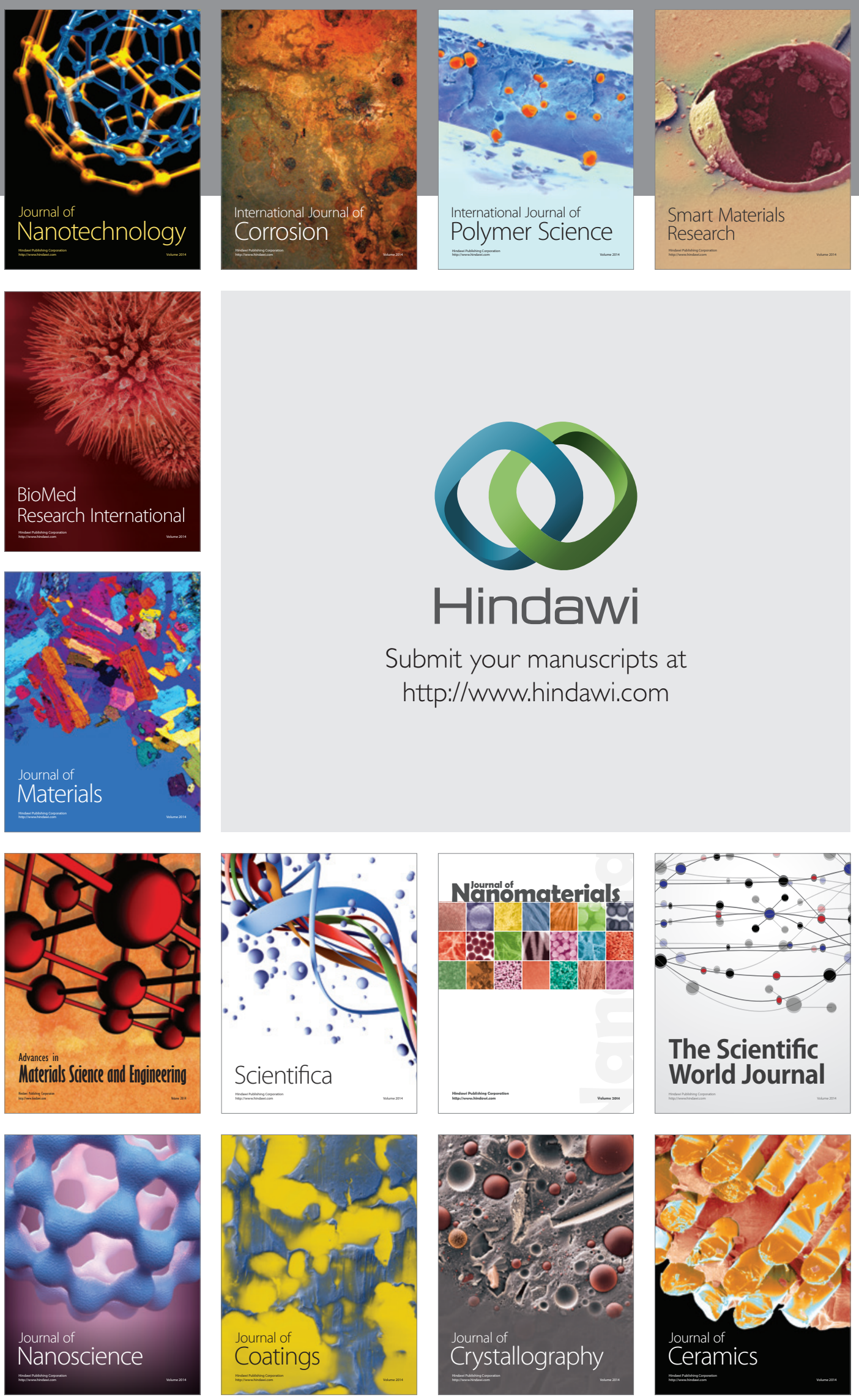

The Scientific World Journal

Submit your manuscripts at

http://www.hindawi.com

\section{World Journal}

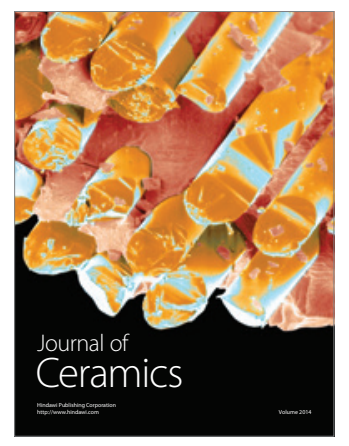

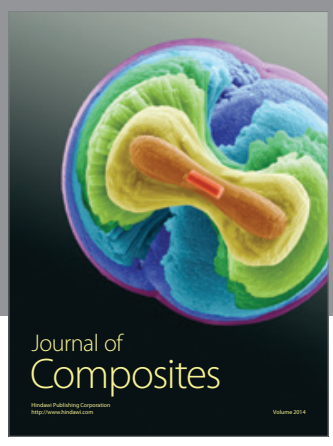
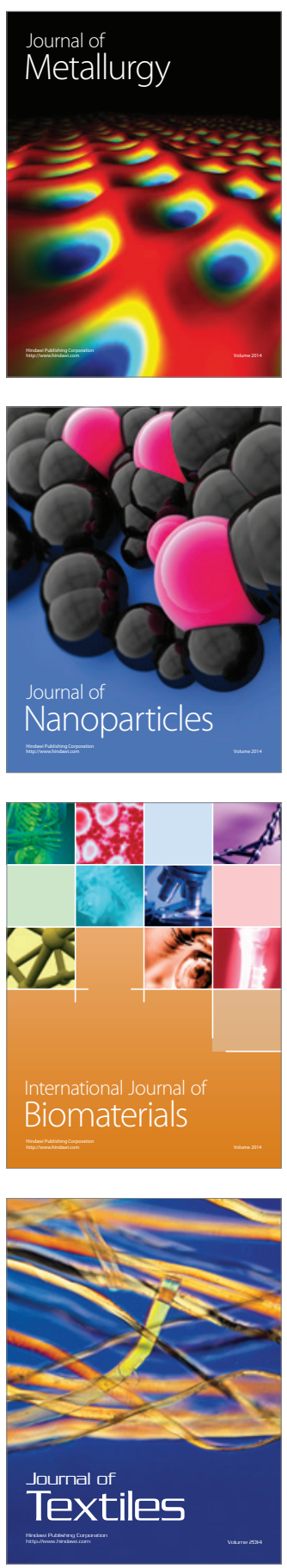